\title{
Enfoques metodológicos en la investigación histórica: cuantitativa, cualitativa y comparativa
}

\author{
Methodological approaches in historical research: \\ quantitative, qualitative and comparative
}

\section{Arturo Alexander Sánchez Molina* Angélica Murillo Garza**}

* Egresado de la Escuela Normal Superior "Profr. Moisés Sáenz Garza", Monterrey, México. Tiene estudios de Licenciatura en Educación Secundaria con Especialidad en Historia; ha realizado tres posgrados: Especialidad en la Enseñanza de las Ciencias Sociales, Maestría en Docencia y Maestría en Enseñanza de la Historia de México.Correo electrónico: alexandermolina9337@gmail.com

(1) https://orcid.org/0000-0001-8348-6883

* Profesora-investigadora en la Escuela Normal Superior "Profr. Moisés Sáenz Garza", Monterrey, México. Es doctora en Ciencias de la Educación; investigadora Perfil PRODEP; ha formado parte de diferentes Consejos Editoriales en revistas de investigación en español, inglés, francés; participante en redes de investigación nacionales e internacionales; es socia de número en la Sociedad Nuevoleonesa de Historia, Geografía y Estadística, AC. Correo electrónico: mes.mle.angelicamg@hotmail.com

https://orcid.org/0000-0002-5708-428X

Historial editorial

Recibido: I2-marzo-202I

Aceptado: I6- junio-202I

Publicado: 30-julio-202I

ISSN-e: 2594-2956 
Enfoques metodológicos en la investigación histórica: cuantitativa, cualitativa y comparativa

\section{Resumen}

El presente escrito tiene la finalidad de dar a conocer al lector las características de los enfoques metodológicos que emplean los historiadores para llevar a cabo procesos de investigación. Existen diferentes caminos para indagar la realidad social. La investigación científica en ciencias sociales se puede abordar desde tres paradigmas o alternativas metodológicas: cuantitativa, cualitativa y comparativa. A pesar de que cada opción metodológica se sustenta en supuestos diferentes y tiene sus reglas y formas básicas de acción, establecidas y compartidas por la propia comunidad científica, no son métodos excluyentes, se complementan. Se pretende mostrar al lector como se puede ejercer la metodología del método comparativo y su paso enfático en el desarrollo de la historia $\mathrm{y}$, por qué no, en su aplicación en el aula para comprender los fenómenos académicos relacionados directamente con la enseñanza y el aprendizaje de la Historia en el nivel de educación básica, específicamente en secundaria.
Palabras
clave:
metodológicos,
Enfoques
histórica, metodología comparativa, metodología cualitativa, metodología cuantitativa.

Methodological approaches in historical research: quantitative, qualitative and comparative

\section{Abstract}

The purpose of this paper is to make the reader aware of the characteristics of the methodological approaches used by historians to carry out research processes. There are different ways to investigate social reality. Scientific research in social sciences is approached from three paradigms or methodological alternatives: quantitative, qualitative, and comparative. Although each approach is based on different assumptions and has its rules and basic forms of action, already established and shared by the scientific community, they do not exclude one another but rather complement each other. This study aims at demonstrating how the methodology of the comparative method and its emphatic step can be applied in the development of history and in the classroom, to understand the academic phenomena directly related to the teaching and learning of History at the basic education level, specifically in secondary school.

Keywords: Methodological approaches, Historical research, Comparative methodology, Qualitative methodology, Quantitative methodology. 
Approches méthodologiques de la recherche historique: quantitative, qualitative et comparative

\section{Résumé}

Le présent mémoire a pour objet de faire connaître au lecteur les caractéristiques des approches méthodologiques utilisées par les historiens pour mener à bien les processus de recherche. Il existe différentes façons d'explorer la réalité sociale. La recherche scientifique en sciences sociales peut être abordée à partir de trois paradigmes ou alternatives méthodologiques: quantitative, qualitative et comparative. Bien que chaque option méthodologique repose sur des hypothèses différentes et ait ses règles et formes fondamentales d'action, établies et partagées par la communauté scientifique elle-même, elles ne sont pas exclusives, elles se complètent. L'objectif est de montrer au lecteur comment la méthodologie de la méthode comparative peut être appliquée et comment et pourquoi elle ne l'est pas, dans son application en classe pour comprendre les phénomènes académiques directement liés à l'enseignement et à l'apprentissage de l'histoire au niveau de l'éducation de base, en particulier au niveau secondaire.

Mots-clés:

méthodologiques,

historique, comparative, qualitative, quantitative.
Podejścia metodologiczne w badaniach historycznych: ilościowe, jakościowe i porównawcze

\section{Streszczenie}

Celem tego artykułu jest uświadomienie czytelnikowi cech metodologicznych stosowanych przez historyków do przeprowadzania procesów badawczych. Istnieją różne sposoby badania rzeczywistości społecznej. Do badań naukowych w naukach społecznych można podchodzić z trzech paradygmatów lub alternatyw metodologicznych: ilościowych, jakościowych i porównawczych. Chociaż każda opcja metodologiczna opiera się na różnych założeniach i ma swoje podstawowe zasady i formy działania, ustanowione i wspólne dla samej społeczności naukowej, nie są one metodami wyłącznymi, uzupełniają się wzajemnie. Ma na celu pokazanie czytelnikowi, w jaki sposób można korzystać z metodologii porównawczej i jej zdecydowanego kroku w rozwoju historii i jej zastosowaniu w klasie, aby zrozumieć zjawiska akademickie bezpośrednio związane z nauczaniem i uczeniem się historii na poziomie edukacji podstawowej, szczególnie w szkołach średnich

Slowa kluczowe: Podejścia metodologiczne, Badania historyczne, Metodologia porównawcza, Metodologia jakościowa, Metodologia ilościowa. 


\section{Introducción}

Este artículo se ha escrito con base en las investigaciones y opiniones de diversos autores que versan sobre la investigación histórica, los enfoques o metodologías cuantitativa, cualitativa y comparativa. Se realiza una aproximación al camino a seguir para recopilar información en la investigación histórica a través de las metodologías referidas, así como el tipo de instrumentos que utilizan para la recolección de datos. Para Hurtado (2008) una revisión documental es una técnica en donde se recolecta información escrita sobre un determinado tema, teniendo como fin proporcionar variables que se relacionan indirectamente o directamente con el tema establecido.

La investigación es un proceso a través del cual se genera conocimiento de la realidad con la intención de explicarla, comprenderla, y transformarla, de acuerdo a las necesidades materiales y socioculturales que cambian vertiginosamente. Como refieren Hernández, Baptista y Fernández (2010) la investigación "Es un conjunto de procesos sistemáticos, críticos y empíricos que se aplican al estudio de un fenómeno" (p. 4). Las metodologías tienen la finalidad de resolver problemas, bajo reglas de validez y aceptación, cobijadas por el método científico. Estas metodologías se pueden adaptar a problemáticas de aprendizaje dentro del aula que, sin duda alguna, pueden solucionarse o disminuirse, al asignar un rol de investigador al docente y a los estudiantes para su aprendizaje.

Existen diferentes caminos para indagar la realidad social. Por un lado, el paradigma cuantitativo y por otro el cualitativo. "La investigación científica en ciencias sociales se puede abordar desde dos paradigmas o alternativas metodológicas: cuantitativa y cualitativa" (Monje, 20II, p. Io). Pero además de estas dos existe una tercera llamada metodología

150 comparativa, aplicada en las ciencias sociales, específicamente en lo político, que surgió en los años sesenta y a la cual Nohlen (1998) define como "un procedimiento de la comparación sistemática de casos de análisis que en su mayoría se aplica con fines de generalización empírica y de la verificación de hipótesis" (p. I). 
A pesar de que cada opción metodológica se sustenta en supuestos diferentes y tiene sus reglas y formas básicas de acción, establecidas y compartidas por la propia comunidad científica, no son métodos excluyentes, se complementan. Este artículo presenta -a su vez- una reflexión acerca de la utilización del método comparativo en estudios cualitativos, en el campo de las ciencias sociales, especialmente de la ciencia política, y muestra al lector como se puede ejercer la metodología del método comparativo y su paso enfático en el desarrollo de la historia y por qué no, en su aplicación en el aula para solucionar y entender los fenómenos académicos relacionados directamente con la enseñanza y el aprendizaje de la Historia en el nivel de educación básica, específicamente en secundaria.

"Las investigaciones históricas permiten conocer y reflexionar sobre un fenómeno, considerando resaltar los conceptos e hipótesis y la comprensión de las relaciones de la Historia con el Tiempo, con la memoria o con el Espacio" (De Almeida, 20I6, p. 383). Para Grajales (2002) la investigación histórica se expresa como "El esfuerzo que se realiza con el propósito de establecer sucesos, ocurrencias o eventos en un ámbito que interesa al historiador; se entiende por metodología el modo como se enfocan los problemas y se buscan las respuestas" (p.7).

¿Cuál es el proceso a seguir para recopilar información en la investigación histórica?

Para recopilar información en la investigación histórica, Grajales (2002) menciona que primero el historiador/investigador debe establecer un problema delimitado. Esto se logra cuando el investigador establece una o varias preguntas específicas frente a un hecho o evento histórico, con el fin de precisar lo sucedido, establecer comparaciones o determinar causas y/o consecuencias. Esto es de suma importancia, porque no se puede investigar un campo amplio ya que se perdería el objetivo de la investigación de una problemática en concreto. Si la metodología se aplica en el aula, se pueden citar, por ejemplo, las dificultades que tienen los alumnos para comprender conceptos relacionados con la cronología, conceptos políticos, o las nociones de 
espacialidad y temporalidad, solo por mencionar algunas que son muy frecuentes al abordar la Historia en las aulas de nivel básico (secundaria).

El historiador/investigador debe además establecer una hipótesis histórica a partir de preguntas de investigación, pues es parte del proceso. Al respecto Grajales (2002) expresa que:

El investigador, fundamentado en su conocimiento de las corrientes interpretativas, la información disponible, los elementos comunes en otros eventos semejantes, si los hay, identifica las respuestas más plausibles a la pregunta de investigación, para luego dedicarse a la tarea de recoger evidencias y datos que permitan comprobar la viabilidad de las hipótesis o su rechazo (p. II).

Como parte fundamental del proceso de investigación histórica, el historiador/investigador debe apoyarse de fuentes primarias y secundarias. Para Grajales (2002) "Corresponde al investigador proveerse de la información necesaria para cuestionar o comprobar sus hipótesis" (p. II). El investigador debe tomar como referencia datos de primera mano, es decir, de las fuentes que le servirán para estudiar el fenómeno o problema y con ello comprobar o rechazar una o varias hipótesis.

\section{Metodología cuantitativa}

De acuerdo con Bonilla y Rodríguez (I997) "La investigación cuantitativa se inspira en el positivismo. Este enfoque investigativo

152 plantea la unidad de la ciencia, es decir la utilización de una metodología única que es la misma de las ciencias exactas y naturales" (p. 83). Dicha metodología usualmente parte de cuerpos teóricos aceptados por la comunidad científica, con base en los cuales formula hipótesis sobre relaciones esperadas entre las variables que forman parte del problema que se estudia. 
Para Monje (20II) la investigación cuantitativa (empírico-positivista) establece una separación tajante entre el sujeto que conoce y la realidad observada, la cual es aprehendida por el investigador como agente externo a la misma. Para esta metodología, la medida y cuantificación de los datos constituye el procedimiento para alcanzar la objetividad en el proceso de conocimiento.

Sobre la investigación cuantitativa, así refiere Monje (20II) "La pretensión explicativa es característica de los estudios cuantitativos, de donde se deriva la predicción, la manipulación técnica y el control sobre los acontecimientos o hechos, preferentemente del mundo natural" (p. I4). La investigación bajo este enfoque tiene mayor preocupación por los procedimientos analíticos, es decir, por la fragmentación y el estudio de las partes que constituyen el todo social. Esta metodología, según Monje (20II), tiene predominio del método hipotético-deductivo y por ello:

Implica que los científicos sociales focalicen su atención en probar hipótesis a partir de un doble referente: el cuerpo conceptual de un lado y la realidad concreta que se estudia del otro lado. El método científico se aplica primordialmente para fundamentar; justificar y respaldar hipótesis específicas que se deducen de un marco conceptual (p. I6).

La estructura para la investigación cuantitativa se integra por las siguientes fases: Teoría, hipótesis, observaciones, recolección de datos, análisis de datos y resultados. Entre la teoría y la hipótesis se da la deducción; entre la hipótesis y la recolección de datos se presenta la operacionalización; entre la recolección y el análisis de datos se suscita el procesamiento de datos; entre el análisis de datos y los resultados emerge la interpretación y, por último, de los resultados se origina la inducción. 


\section{Metodología cualitativa}

Con relación a esta metodología, Monje (20II) menciona que "Se nutre de la hermenéutica, la fenomenología y el interaccionismo simbólico. El pensamiento hermenéutico parte del supuesto que los actores sociales no son meros objetos de estudio, como si fuesen cosas, sino que también significan, hablan, son reflexivos" (p. I3).

Dicha metodología se interesa por captar la realidad social a través de la mirada de la gente que está siendo estudiada, es decir, a partir de la percepción que tiene el sujeto de su propio contexto. Plantea que la realidad no es exterior a quien la examina, existiendo una relación estrecha entre el sujeto y el objeto de conocimiento. Como refiere Monje (20II) la metodología "Muestra mayor tendencia al examinar el sujeto en su interacción con el entorno al cual pertenece" (p. I4). Con ella se observa mayor interés por lo datos cualitativos, por la indagación más amplia de actitudes, valores, opiniones, percepciones, creencias y preferencias de los sujetos. En la investigación cualitativa, los objetivos pueden orientarse a la descripción, la explicación o la comprensión.

Para Monje (20II) la investigación cualitativa:

Intenta hacer una aproximación global de las situaciones sociales para explorarlas, describirlas y comprenderlas de manera inductiva. Es decir, a partir de los conocimientos que tienen las diferentes personas involucradas en ellas y no deductivamente, con base en hipótesis formuladas por el investigador externo (p. I6).

En esta metodología no se configura un marco fijo e inmodificable, sino un punto de referencia que indica qué se va a explorar (objetivos), cómo

154 debe procederse (la estrategia) y qué técnicas se van a utilizar (la recolección). "Aunque se aplica un esquema abierto de indagación, se va refinando, puntualizando o ampliando según lo que el investigador vaya comprendiendo de la situación, el proceso debe iniciarse con un plan de trabajo referencial" (Bonilla y Rodríguez, I997, p. 125). 
Como bien afirma Monje (20II) "El proceso de la investigación cualitativa, se plantea, por un lado, que observadores competentes y cualificados pueden informar con objetividad, claridad y precisión acerca de sus propias observaciones del mundo social, así como de las experiencias de los demás" (p. 32). El proceso a seguir en la metodología cualitativa es definir una situación problema, explorar esa situación y elaborar un plan de acción, posteriormente, realizar trabajo de campo donde se recolectan datos cualitativos y se organiza la información y, por último, se identifican los patrones culturales al analizar e interpretar la información obtenida, para establecer una conceptualización inductiva.

No hay un único método a través del cual podamos alcanzar y dominar el desarrollo y la experiencia, como consecuencia, los investigadores despliegan una multitud de métodos capaces de llegar al objeto de estudio. De esta forma Denzin y Lincoln (I994) mencionan que cada investigador se enfrenta al mundo desde un conjunto de ideas, un marco (teoría) que determina una serie de cuestiones (epistemología) que son examinadas de una forma determinada (metodología, análisis).

\section{Metodología comparativa}

La metodología comparativa es el procedimiento de la comparación sistemática de casos de análisis que en su mayoría se aplica con fines de generalización empírica y de la verificación de hipótesis. Cuenta con una larga tradición en la metodología de las ciencias sociales; ciencias políticas. Respecto al método comparativo, Nohlen (2003) refiere que "Se entiende exactamente en los tratados metodológicos y en la práctica de investigación: entre otros factores, es una consecuencia de que el concepto muchas veces se emplea de manera sinónima con comparación: análisis comparativo e investigación comparativa" (p.30).

A esta comparación cualitativa se opone la cuantitativa que utiliza procedimientos estadísticos, la comparación es inherente a cualquier procedimiento científico, el método científico es inevitablemente comparativo y toda política es de alguna manera política comparativa, 
de esta perspectiva se deriva que la idea de un método comparativo autónomo que parece resultar redundante, en las ciencias sociales deben igualarse el método comparativo y el método científico.

La comparación es el criterio de la interpretación valorativa de los resultados empíricos. Así refiere Álvarez, (20II) "no es el principio del conocimiento científico -como éste debe considerarse más bien el postulado del examen crítico de teorías-, permite la evaluación comparativa de los resultados empíricos" (p. 240). El método comparativo, en sentido estricto, busca examinar nexos causales y trata de aislar los factores que pueden ser considerados como causa variable independiente de un efecto variable dependiente que dé como resultado una causalidad.

La reflexión metodológica se refiere primordialmente a su variante cualitativa, ya que en este caso es casi imposible una estandarización del método comparativo porque típicamente este se aplica sobre todo allí donde los métodos estandarizados de las ciencias sociales no funcionan debido a lo específico del objeto a analizar, ya sea porque el referido no puede ser examinado de manera experimental o porque el número de casos es tan escaso que no pueden utilizarse procedimientos estadísticos. En la aplicación de la metodología cualitativa "hay tres métodos propios de las ciencias sociales: el experimento, el método estadístico y el estudio de caso" (Nohlen, 2003, p. 40).

\section{El tipo de instrumentos que se utilizan para recopilar datos}

Existen diferentes instrumentos de los que se apoyan los investigadores para la recogida de datos para su posterior análisis y sistematización en

156 el informe. Como bien señala Monje (20II) "Toda la información obtenida se va a concentrar en los instrumentos que el historiador/investigador utiliza para recolectarla y registrarla" (p. 25). Algunos instrumentos que sugiere el autor son los formularios, las pautas o guías de observación, las pruebas psicológicas, las escalas de opiniones y actitudes, así como listas de control, entre otros. 
De acuerdo con Monje (20II) “La recolección de datos se efectúa mediante la aplicación de instrumentos diseñados en la metodología, utilizando diferentes métodos como la observación, la entrevista, la encuesta, los cuestionarios, los test, la recopilación documental y otros" (p. 28). Los positivistas buscan las causas del problema del que parte la investigación histórica mediante instrumentos tales como cuestionarios, inventarios o estudios demográficos que producen datos susceptibles de análisis estadístico.

Para Monje (20II) los métodos de recolección estructurada de datos cuantitativos reúnen información que se cuantifica con mayor facilidad, aunque también se puede cuantificar la información no estructurada. Destacan las técnicas de la entrevista estructurada, el cuestionario auto diligenciado, la observación sistemática, regulada o controlada, así como las escalas de actitud y opinión.

La entrevista es un método diseñado para obtener respuestas verbales a situaciones directas o telefónicas, entre el entrevistador y el encuestado. El instrumento entrevista estructurada lo describe Monje (20II) como "La que emplea un cuestionario (o guion de entrevista) con el objeto de asegurarse que a todos los encuestados se les hacen las preguntas de manera estandarizada, esto es, de igual modo y en el mismo orden" (p. I36).

El método de encuesta resulta adecuado para estudiar cualquier hecho o característica que las personas estén dispuestas a informar. Su utilización, menciona Monje (20II) que "Se puede asumir bajo distintos enfoques: investigaciones descriptivas; investigaciones comparativas y evaluativas complementadas, los tipos de encuestas van de ser descriptivas: orientadas explicativas, seccionales o transversales y longitudinales: retrospectivos prospectivos" (p. 134).

Para la elaboración del instrumento es importante redactar el método de encuesta: es individualista, estático. Trabajar con formalidades estadísticas, etapas de encuesta: determinación de que el método de encuesta es adecuado y la formulación de objetivos: generales y específicos. Determinación de la cobertura de la encuesta; personas, lugar y tiempo. 
Es importante la elaboración de un cuestionario autodiligenciado, que es un formato resuelto en forma escrita por los propios sujetos de la investigación, reduce los sesgos ocasionados por la presencia del entrevistador, esto "Facilita el análisis y reduce los costos de aplicación, sus desventajas el encuestador pierde el control de la secuencia de las preguntas y presenta menor porcentaje de respuestas" (Álvarez, 20II, p. I37).

Para el historiador/investigador positivista, las herramientas o instrumentos para realizar las investigaciones y analizar y comprobar los hechos históricos, son las fuentes reales, es decir, las fuentes primarias y secundarias, puesto que para obtener el conocimiento histórico el historiador "Necesita de la utilización de fuentes, ya sean documentos, testimonios u objetos. $\mathrm{Su}$ argumentación se basa en la interpretación de éstas" (Hurtado, s./f, p. I). Debe recopilar toda la información histórica de fuentes confiables para comprobar su hipótesis, pues esa es su materia prima.

Se entiende por fuente de información toda persona $u$ objeto que disponga u ofrezca datos relevantes respecto al asunto en investigación. "Estas fuentes pueden ser clasificadas en dos categorías básicas: fuentes primarias y fuentes secundarias" (Grajales, 2002, p.II). Como se mencionó con antelación, la materia prima de los historiadores son las fuentes de información histórica (primarias y secundarias), así como las metodologías de investigación y los instrumentos para recopilar datos.

Respecto a la fuente primaria, Grajales (2002) la define como "Aquella que está directamente relacionada en términos de tiempo y espacio con el evento, hecho, suceso u ocurrencia que se estudia. Puede ser el testimonio de personas que participaron en el hecho o lo observaron directamente..." (p. II). De igual manera, Hurtado (s./f.) define a las

158 fuentes primarias como "Los documentos, testimonios $u$ objetos originales que le permiten al historiador investigar directamente en ellos, sin la intervención de un intermediario" (p. 5).

Para Grajales (2002) las fuentes primarias pueden ser testimonios orales que relaten su experiencia del pasado, documentos elaborados con el propósito expreso de transmitir información que pueda ser usada en el 
futuro y reliquias o testimonios históricos cuya existencia no tuvo como objeto deliberado y consciente transmitir información para ser utilizada en el futuro.

Sobre esta misma idea, el quehacer del historiador/investigador con las fuentes primarias, es decir, el procedimiento que sigue utilizar fuentes primarias (escritas, iconográficas y orales) según expresa Hurtado (s./f.) es el siguiente:

Busca, encuentra, selecciona, clasifica, edita cuando es necesario, contrasta, para obtener no sólo información, sino orientación a su investigación; pero, durante todo este proceso interpreta, argumenta, reflexiona y valora las fuentes a partir de una intencionalidad y tomando en cuenta las circunstancias ( $p$. 4).

La fuente secundaria que le sirve al historiador para recopilar datos tiene ciertas características que pueden restarle objetividad, puesto que "Tiene en general un valor limitado por causa de las distorsiones que sufre la información al pasar de un emisor a un receptor" (Grajales, 2002, p. I2).

Es ahí donde el investigador puede interpretar mal el mensaje y distorsionar la información y discrepar con la idea original del autor de la fuente en su contexto. También el autor del artículo expresa que la persona que escribe o provee una información de segunda mano no se halla en la escena del acontecimiento que se estudia en el momento cuando acontece; por lo general relata lo que otro, que dice haber estado allí, dijo o escribió. Esto ocasiona que muchas veces las interpretaciones se alteren.

Las fuentes secundarias se pueden describir como "Los resultados concretos de la utilización de las fuentes primarias, es decir, libros, ensayos, artículos, biografías, monografías, entre otros. Este tipo de fuentes no son de poco valor, pues son el producto de años de investigación histórica" (Hurtado, s/f, p. 6). Las fuentes secundarias pueden ser escritos (libros, revistas, periódicos, entre otros), auditivos 
(grabaciones de conferencias) y auditivos y visuales (teleconferencias, videoclips).

El uso de fuentes históricas, de las cuales los historiadores/investigadores recopilan datos, se manifiestan tres aspectos, que según Hurtado (s./f.) se describen así:

La utilización de fuentes en historia tiene tres aspectos: primero, como base desde la que se construye el conocimiento histórico (fundamentación); segundo, como medio por el que se transita para construir dicho conocimiento (demostración); y tercero, como límite, en el sentido de que pone una frontera al historiador, impidiéndole que pueda elucubrar sin argumentos acerca de su tema de investigación (verificación) (p. I).

El autor hace énfasis en que los tres aspectos son tan importantes y se debe desarrollar adecuadamente, así como ser lo más objetivos en la interpretación de las fuentes y no salirse de contexto, además de identificar, una vez concluida la investigación histórica, la confiabilidad necesaria y la suficiente certeza de que los resultados son producto de una investigación seria y científica.

Como se sabe, cada historiador/investigador realiza las interpretaciones para construir el conocimiento histórico, producto de sus reflexiones. "Las fuentes son las mismas, no cambian, pero la mirada del historiador no es la misma" (Hurtado, s./f, p. 2) Aun así, debe propiciar siempre la objetividad, misma que se puede definir como "La no intervención de cualquier elemento afectivo en la relación que se establece entre el observador y lo observado" (Monje, 20II, p. I5).

Es importante la labor del historiador/investigador para recopilar datos

160 a través de las fuentes, obtener el conocimiento histórico, validar o rechazar sus hipótesis respecto a un problema o hecho histórico y elaborar el discurso histórico con sentido y objetividad. El historiador "Ordena, reordena, edifica, construye, interpreta, reinterpreta, resuelve, conecta, le da sentido a lo material para crear una nueva idea a partir de la utilización de fuentes" (Hurtado, s./f, p. 3). 


\section{Las técnicas para el análisis de la información}

"Las técnicas e instrumentos que utilizará cada perspectiva paradigmática serán muy similares, pero diferirán en el grado de abstracción, su enfoque o sus resultados" (Monje, 20II, p. 37). Después de clasificar las fuentes (primarias y secundarias) otro aspecto que es de suma importancia en el proceso de la investigación histórica que llevan a cabo los historiadores/investigadores es criticar dichas fuentes. Esta acción es realizada con la finalidad de validar o rechazar la o las hipótesis planteadas. Entonces, el investigador tiene "La tarea de determinar la calidad y relevancia de la información que la fuente provee. Los datos deben ser sujetos a un análisis cuidadoso a fin de discriminar entre lo falso y lo verdadero" (Grajales, 2002, p. 12). Además, refiere que no existe garantía de que a través del criticismo cualquier evento del pasado pueda ser probado totalmente.

El proceso para evaluar y juzgar las fuentes que recopilan los historiadores/investigadores para la investigación histórica bajo una metodología se denomina crítica. Como parte del proceso de la investigación, Grajales (2002) menciona que "Es mediante el proceso de crítica o juicio de la fuente que el historiador determina las evidencias históricas en las cuales se apoyará para interpretar o comprobar sus hipótesis" (p. 12). Los instrumentos cualitativos, en comparación con los cuantitativos, enfatizan los estudios que emplean métodos de recolección que difieren de aquellos que se utilizan en estudios cuantitativos. "Sin desconocer la naturaleza de cada problema investigativo, es aconsejable combinar los métodos de recolección de datos, tanto cuantitativos como cualitativos, para obtener una comprensión más integral de la conducta humana" (Monje, 20II, p. I50).

Los instrumentos cualitativos se podrían identificar de la siguiente manera: I. La Entrevista no estructurada y entrevista dirigida, 2. La entrevista en profundidad, 3. Los grupos focales, 4. La observación simple, no regulada y participante de vida, 5 . Los análisis de contenido y 6. El método etnográfico.

La entrevista no estructurada es flexible y abierta, se procede sin un concepto preconcebido del contenido o flujo de información que se 
desea obtener, aunque los objetivos de la investigación rigen las preguntas. "El contenido, orden, profundidad y formulación se hallan sujetos al criterio del entrevistador" (Álvarez, 20II, p. I52).

Si bien el investigador, sobre la base del problema, los objetivos y las variables, elabora las preguntas antes de realizar la entrevista, modifica el orden, la forma de encauzar las preguntas o su formulación para adaptarlas a las diversas situaciones y características particulares de los sujetos de estudio. Las entrevistas comparten una estructura básica en la que el investigador tiene las preguntas y el sujeto da las respuestas. Sin embargo, "Las entrevistas cualitativas, a diferencia de las estructuradas, siguen el modelo de una conversación entre iguales, donde el propio investigador es el instrumento y no lo que está escrito en el papel" (Álvarez, 20II, p. I53).

Se distinguen tres tipos de entrevistas en profundidad: la primera es la historia de vida, en que el investigador trata de obtener experiencias destacadas de la vida del entrevistado; el segundo: pretende lograr un aprendizaje sobre acontecimientos y el tercer tipo, pretende proporcionar un cuadro amplio de escenarios, situaciones o personas, manteniendo en común las otras características.

"El grupo focal también se denomina "entrevista exploratoria grupal", donde un grupo reducido (de seis a doce personas) y con la guía de un moderador, se expresa de manera libre y espontánea sobre una temática" (Álvarez, 20II, p. I55). La técnica de los grupos focales es una reunión con modalidad de entrevista grupal abierta y estructurada, el objetivo fundamental del grupo focal es alcanzar o lograr el descubrimiento de una estructura de sentido compartida sobre un aspecto particular de interés, en este tipo de entrevista se reúnen de Io a I5 individuos para discutir un tema y el entrevistador que los conduce

162 formula preguntas sobre el tema a tratar.

En la observación simple, no regulada o no controlada, sólo se tienen unos lineamientos generales para la observación sobre aspectos del fenómeno que el investigador tiene interés en conocer. En la observación participante el investigador o el responsable de recolectar los datos se involucra directamente con la actividad objeto de la 
observación. "El objetivo de la observación en la perspectiva cualitativa es comprender el comportamiento y las experiencias de las personas como ocurren en su medio natural. Personas en sus medios con un mínimo de estructuras y sin interferencia del investigador" (Álvarez, 20II, p. I55). Se considera como una técnica que permite el registro de las acciones perceptibles en el contexto natural y la descripción de una cultura desde el punto de vista de sus participantes. La observación participativa se considera como una técnica que permite el registro de las acciones perceptibles en el contexto natural y la descripción de una cultura desde el punto de vista de sus participantes.

La crítica formulada a este método señala la subjetividad del investigador, como factor que puede sesgar el análisis. Así mismo, Álvarez (20II) menciona que "La cientificidad del método se logra mediante: la transparencia del investigador, es decir, llevando sistemáticamente y de la manera más completa e imparcial sus notas de campo" (p. I54).

Las historias de vida son revelaciones narrativas acerca de la vida de la persona y se emplean con frecuencia para estudiar patrones culturales en el caso de las ciencias sociales. La cientificidad del método se incrementa apelando a materiales objetivos, como los documentos de archivo, el conocimiento del contexto socioeconómico y de los acontecimientos locales de la época. "También son importantes las críticas de autoridad, de identidad, de originalidad y de restitución" (Álvarez, 20II, p. I55).

La entrevista es un reporte verbal producto de la interacción entre el investigador y el sujeto investigado en el cual este último aporta información primaria acerca de su conducta, de experiencias a las cuales ha estado expuesto o ha participado. La entrevista etnográfica tiene como objetivo registrar la manera como el entrevistado construye su realidad y experiencia; averiguar lo que otra persona piensa o siente con respecto a una situación particular.

Las técnicas cualitativas se orientan hacia la entrevista abierta que permite al entrevistado responder de acuerdo con su experiencia personal en sus propias palabras. 
Las entrevistas abiertas difieren marcadamente de los cuestionarios de preguntas cerradas que incluyen alternativas limitadas de respuesta, los cuales son más adecuados para la investigación por encuesta (Álvarez, 2OII).

El registro de la información etnográfica se efectúa corrientemente en diarios de campo, cuadernos de notas, dibujos, croquis, grabaciones, fotografías, filmaciones. El diario de campo "Es uno de los instrumentos más importantes de registro” (Álvarez, 20II, p. I67). Puede ser cualquier libro, libreta, cuaderno o agenda de anotaciones, en donde se lleva un registro cronológico de los principales acontecimientos que el investigador está presenciando durante el trabajo de campo"

Sobre el papel del etnógrafo, para la gran mayoría de los autores, "es el principal instrumento de investigación. De él depende la selección de la temática a investigar, la filosofía que se adopte en el estudio, el acceso al campo, las relaciones con los sujetos estudiados" (Álvarez, 2008, p. 4). Esto significa que por sobre los medios de registro de la información se halla el propio investigador. "La utilización de diarios de campo, cuadernos de notas, fotografías, grabaciones, dibujos, croquis, objetos de cultura material, etc., están influidos directamente por los propósitos que se les asigne y por el estilo personal de quien los aplica" (Álvarez, 20II, p. I66).

La monografía etnográfica es uno de los medios más importantes para la comunicación de resultados. Esta contiene los aspectos más significativos de la cultura y sociedad estudiada. Álvarez, (2OII) expresa que la estrategia narrativa "Se refiere a las formas de presentación de los datos etnográficos en sus géneros básicos: la monografía y el ensayo" (p. 166). La elaboración de instrumentos para la recolección de datos exige analizar la forma como dicho instrumento de medición cumple 164 con la función para la cual ha sido diseñado.

La confiabilidad se refiere a la capacidad del instrumento para arrojar datos o mediciones que correspondan a la realidad que se pretende conocer, o sea, la exactitud de la medición, así como a la consistencia o estabilidad de la medición en diferentes momentos. A mayor 
confiabilidad de un instrumento, menor cantidad de error presente en los puntajes obtenidos.

La validez se refiere al grado en que un instrumento mide lo que se pretende medir. La forma de garantizar la validez de un instrumento es construirlo una vez que las variables han sido claramente especificadas y definidas. "Todo instrumento debe ser probado en una situación real antes de su aplicación definitiva con el fin de identificar errores o evaluar el tiempo necesario para aplicarlo" (Álvarez, 20II, p. I67).

La eficiencia de las diferentes técnicas depende de lo eficientes que sean para solucionar el problema que representa el análisis de la complejidad causal. "El investigador que se sirve de los instrumentos teóricos preparados para las ciencias sociales, debe evaluar, en el empleo de una abstracción si la complejidad del elemento preseleccionado es adecuada a la complejidad del fenómeno considerado" (Tonon, 20II, p. I5).

En las Ciencias Sociales, el método comparativo utiliza al mismo investigador para dar la precisión y certeza causal del método experimental que se considera el ideal metodológico. Sin embargo, los investigadores sociales pocas veces se acercan a este ideal debido a impedimentos éticos y prácticos. La propuesta se orienta hacia la comparación, que implica contrastes basados en la misma lógica del método experimental.

"Una de las ventajas del método comparativo reside en que deja al investigador mucha libertad para el desarrollo de un diseño propio de investigación adecuado a la situación. Él sólo tiene que aprender a dominar el juego con la concordancia y la diferencia" (De León y De la Garza, 2013, p. 20). Los contextos homogéneos y heterogéneos, los supuestos de contexto estrictos de los métodos de diferencia. Así lo mencionan De León y De la Garza (2013) "En la forma flexible, el investigador decide sobre la selección de las características que presenten los casos incluidos en la comparación, con base en los cuales considera suficiente la similitud o disimilitud de las variables de contexto para aplicar..." (p. 23). 
Los métodos de concordancia y de diferencia en sentido estricto suponen la neutralidad total del contexto, investigaciones en las que se estudian los efectos de la misma variable independiente en dos contextos diferentes pero homogéneos contemplándolos independientemente uno del otro diferente. Es decir, si se supone que el contexto sea homogéneo, no se está deduciendo que no tenga ninguna importancia para el nexo causal hipotético de las variables operativas. De acuerdo con De León y De la Garza (2013):

Las estrategias de investigación del método comparativo en los requisitos estrictos formulados se acercan tanto al experimento que para ellas también aplica lo que para el experimento: que no es aplicable al objeto de las ciencias del espíritu y sociales. Los enfoques estrictos siguen la idea de causalidad lineal (p. I4).

La pregunta esencial se refiere a las diferentes causas de estas diferencias y a su ponderación. Tan sólo el hecho de suponer una multicausalidad y de verificar su existencia significa que se está siguiendo un entendimiento flexible de las estrategias de investigación.

El método comparativo es una herramienta fundamental ya que ayuda a verificar las hipótesis propuestas en la medida en que ofrece una clasificación entre los sujetos de la muestra, así como lo señala León (2013) "Sus respectivas variables y esto genera la oportunidad de poder formular nuevas hipótesis y generar nueva teoría que ayude proponer nuevas alternativas de solución" (p. I4). Respecto al método comparativo, Nohlen (2003) señala que:

Resulta ser reto interesante al ofrecer al investigador un modo flexible pero riguroso de analizar una realidad empírica, con pocos casos y muchas variables. A través de este enfoque se procuró reformular la propuesta de comparación, pasando de la mera descripción y análisis de los hechos al análisis del sentido histórico de los hechos (p. I5).

El método comparativo es una totalidad histórica puesto que el método en cuanto instrumento de conocimiento es el resultado de un proceso sociohistórico de construcción. Tal proceso no consiste simplemente en 
una invención y diseño de técnicas de recolección y análisis de datos, sino que es mucho más amplio y complejo. "La construcción de un método no puede reducirse al diseño de procedimientos, la elaboración de normas y la especificación de sus fundamentos lógicos" (Nohlen, 2003, p. I6).

\section{Una aproximación a la enseñanza y el aprendizaje de la Historia en la escuela secundaria}

En el siglo pasado y en los primeros años del siglo XXI, la enseñanza de la historia se efectuaba de manera tradicional, y solo se promovía la memorización de nombres de personajes, hazañas y fechas. Para los años posteriores se promovió una enseñanza renovada, que potenciara otras habilidades y competencias, con aplicación en el contexto de los estudiantes. Al respecto, Salazar (200I) menciona que el maestro en el aula:

Debe poner en cuestionamiento el paradigma de la enseñanza de la escuela tradicional, según el cual el aprendizaje es un gran depósito de nombres, fechas y batallas que hay que memorizar, con la idea de crear situaciones de enseñanza alternativas y posibles dentro de los márgenes de acción o de autonomía (p. 59).

A partir de los comienzos de la segunda década del siglo XXI, se reformaron los planes y programas de educación básica y se dio paso a una educación basada en competencias y la enseñanza de la historia bajo un enfoque formativo, mismo que la Secretaría de Educación Pública (SEP, 2OIIb) describe como "Una historia que implica evitar privilegiar la memorización de nombres y fechas, para dar prioridad a la comprensión temporal y espacial de sucesos y procesos" (p. 15). No obstante, esto se puede lograr solo a través del estudio de las fuentes, para interpretar el pasado y comprender la realidad actual. Con el modelo de enseñanza tradicional, no era posible involucrar a los estudiantes en la investigación del pasado, ni tampoco promover el desarrollo de habilidades y competencias históricas, que dieran luz a la 
comprensión del presente y del propio desarrollo del pensamiento histórico de los estudiantes.

El estudio de las Ciencias Sociales, en toda su complejidad, supone el uso del pensamiento abstracto formal al más alto nivel que será alcanzado, según explica la psicología evolutiva del desarrollo humano de Piaget, a la edad de I2 años como período de desarrollo evolutivo que dará paso de la inteligencia concreta a la inteligencia abstracta, desarrollada ya en su plena potencialidad. Así, los principales teóricos en psicología evolutiva coincidirán en la necesidad de implantar el aprendizaje significativo desde los primeros niveles educativos. Para Lev Vygotsky (fundador de la psicología histórico-cultural), "El proceso de aprendizaje se produce en una zona llamada de desarrollo próximo, esto es, para que un niño aprenda debe partir de lo que ya sabe, del nivel de desarrollo cognitivo y conductual en el que se encuentra, para, aceptando un desafío que le plantea un determinado problema, avanzar hacia una etapa posterior más enriquecida y más compleja" (citado por García, 2019, p. 29).

El aprendizaje significativo tendrá como finalidad el que los alumnos no se limiten a aprender pasivamente un conocimiento ya elaborado sino que sean los auténticos protagonistas de su propio aprendizaje siguiendo el camino trazado por el profesorado y contando con toda la información, materiales y recursos preparados ad hoc (García, 2019, p. 30).

El aprendizaje significativo potencializa el desarrollo de herramientas y habilidades de investigación seleccionada y conduce a la concreción de aplicaciones para la resolución de problemas de cognitivos para una evaluación eficaz en competencias u objetivos didácticos de la programación que medirán los conocimientos, habilidades y actitudes

168 del alumno en diferentes contextos.

La enseñanza de la Historia es importante puesto que permite a los estudiantes analizar el pasado, entender el presente, su realidad y el devenir histórico. En ese sentido, Moradieros mencionado en Prats y Santacana (20II) señala que: 
El aprendizaje de la Historia supone una reflexión sobre el conjunto de las sociedades en tiempos pasados con el objetivo de enseñar a comprender cuáles son las claves que residen detrás de los hechos, de los fenómenos históricos y de los procesos que explican lo ocurrido y sus causas (p. I3).

Es tan importante enseñar Historia en secundaria además de favorecer aprendizajes y competencias que tienen un potencial formativo.

Los planes de estudios y los programas de Historia del 2011 y 20I7, están diseñados bajo un enfoque competencial, es decir, pretenden potenciar en los estudiantes determinadas habilidades y/o capacidades para movilizar saberes, actitudes y valores en el proceso de enseñanzaaprendizaje de la Historia. Así refieren Gómez, Miralles y Molina (20I5):

Formar al alumnado con base en competencias supone dotarlos de una habilidad específica para interpretar y conferir nuevos sentidos a la realidad sobre la que actúan. Pero también, el profesorado debe tener muy claro el modelo cognitivo de aprendizaje de la materia - en este caso, qué significa que sus alumnos adquieran conocimiento sobre historia-, su relación con las competencias, los instrumentos, o las tareas que permitan obtener información sobre esa adquisición de conocimientos, y una serie de herramientas que permitan interpretarla (p. I2).

Es preciso destacar que las competencias para la Historia que se pretenden desarrollar en la escuela secundaria, según los planes y programas vigentes, son tres: "Comprensión del tiempo y del espacio históricos", "Manejo de la Información Histórica” y "Formación de una conciencia Histórica". Este referente es el indicativo para orientar el quehacer pedagógico y didáctico en función de las tres competencias mencionadas, pero también en función de los fines generales de la historia como materia educativa. Como bien señala Prats (20II) "Los libros incorporan fines y objetivos educativos con especial acento en alguna de las posibilidades que ofrece el trabajar con esta materia en las aulas" (p. 2I). 
La construcción de la historia o interpretación de la misma, se logra a través de la indagación de fuentes primarias y secundarias, que se deben escudriñar, analizar, comparar, hacer inferencias, darles una interpretación contextualizada y objetiva para movilizar conocimientos previos y nuevos, propiciar las habilidades mentales y las destrezas para organizar toda la información, siguiendo un método basada en el método científico de las ciencias sociales, para enseñar a los alumnos que con base en ella se puede entender el presente y su realidad. Estas habilidades que se han externado se derivan de la competencia para el manejo de la información histórica y se deben explotar con apoyo de una metodología de aprendizaje por descubrimiento, la cual parte del método científico.

El desarrollo de la competencia relacionada con aprender a manejar información requiere "Identificar lo que se necesita saber; aprender a buscar; identificar, evaluar, seleccionar, organizar y sistematizar información; apropiarse de la información de manera crítica, utilizar y compartir información con sentido ético" (SEP, 20IIa, p. 38).

En términos didácticos, la competencia para el manejo de la información, según Arista y Reynoso (20I7) se define como:

El conjunto de conocimientos, habilidades y actitudes que el estudiante debe poner en práctica para identificar lo que necesita saber en un momento dado, buscar efectivamente la información que esto requiere, determinar si esa información es pertinente para responder a sus necesidades y finalmente convertirla en conocimiento útil para solucionar problemas de información en contextos variados y reales de la vida cotidiana (p. 26).

170 Para promover la competencia del manejo de la información histórica en el aula, "Hay que pugnar por una historia problema, abierta al desarrollo de problemas, que encaucen el interés del alumno, que vaya más allá de los acontecimientos y logre meter en "problemas cognitivos a los alumnos" (Salazar, 200I, p. 84). Esto promoverá que favorezcan las habilidades para la búsqueda, el manejo de la información histórica, y 
con los hallazgos derivar en la comprensión del presente, y un conocimiento construido que podrá ser difundido.

En el método de las ciencias sociales se investiga con base en planteamientos, es decir se problematiza un tema histórico. Sobre esto, Arista y Reynoso (2017) refieren que las preguntas sólo pueden responderse "Con el desarrollo de habilidades para obtener otro nivel o tipo de conocimientos, después de accionar los sentidos para determinar cuál es la información que permite resolver la situación que dio pie a la búsqueda" (p. I5).

De acuerdo con el currículum oficial se establece como meta educativa que los egresados de secundaria posean habilidades para el manejo de información histórica, en el fondo señala que aquellos deben aprender el método de la ciencia histórica como procedimiento para adquirir el conocimiento histórico y al mismo tiempo desarrollar las habilidades del pensamiento científico. Es por ello que de las bondades de trabajar con las fuentes es favorecer la propia interpretación de la historia, claro, delimitada en un periodo y en un hecho o proceso histórico del que parte la problematización, a manera de investigación especializada de la Historia. "El trabajo directo con fuentes históricas favorece el desarrollo de la competencia de la interpretación histórica, pero también la incorporación de la experiencia histórica" (Santisteban, 20Io, p. 49).

Santisteban mencionado en Arista y Reynoso (2017), con relación a la didáctica de la historia que "El hecho de que el alumno trabaje con las fuentes históricas le permite aprender e interpretar, comparar, clasificar y dar respuestas a interrogantes a partir de las fuentes históricas" (p. I6). Es de esta manera que la competencia para el manejo de información histórica se potencia, ya los estudiantes podrán saber qué y cuáles son las fuentes históricas, buscarlas, curarlas, leerlas, interpretarlas y utilizar esa información o conocimiento para beneficio propio o de la colectividad en la que se participa.

Sobre el manejo de las fuentes, que proveen información histórica, Agüera (20I4) menciona que "Son indispensables para el desarrollo del pensamiento crítico. Debemos de utilizar diversos recursos didácticos 
para ayudar a desarrollar en el alumnado una asimilación y una comprensión de los discursos y una afectividad hacia los mismos" (p. 37). Entonces, no basta con solo tener acceso a las fuentes históricas y proporcionarlas a los estudiantes, sino que se debe tratar la información y lograr acceder al conocimiento del pasado, para resolver un problema o interpretarlo y exponer los hallazgos y las conclusiones sobre él.

Es importante dotar al alumno de herramientas, pero principalmente explicarle cómo se puede acceder al pasado y reconstruirlo en el presente, con base en un problema del presente, que lo llevará al pasado a través del método científico. "El profesor debe de convertirse en mediador para guiar al alumno investigador, para que éste no solamente sea un espectador del discurso histórico, sino que lo construya" (Agüera, 20I4, p. 37). Para acceder al pasado, a través del manejo de la información histórica, se puede empezar con el método por descubrimiento, pero también con el método regresivo, los cuales han tenido gran éxito y relevancia en las aulas de la escuela secundaria, desde el primer grado hasta el tercero.

"Un buen maestro, partiendo del punto en el que encuentra a sus estudiantes, tiene la tarea de llevarlos lo más lejos posible en la construcción de los conocimientos planteados en los planes y programas de estudio y en el desarrollo de su máximo potencial" (SEP, 20I7, p. II6). En ese sentido, es importante tener conocimiento de métodos, técnicas y estrategias para contribuir a que los estudiantes aprendan a pensar históricamente y a construir su propio conocimiento histórico; apoyándose de los métodos que sugieren Joaquín Prats y Joan Santacana, para la enseñanza-aprendizaje de la Historia.

La metodología de aprendizaje por descubrimiento permite acercar a los estudiantes a la investigación histórica de fuentes primarias y 172 secundarias, eliminando ese método que la mayoría de los docentes usan en las aulas en el proceso de enseñanza-aprendizaje, denominado método discursivo. Con ello, los alumnos no desarrollan sus habilidades y competencias históricas, puesto que solo logran el aprendizaje por recepción con base en todo lo que los docentes les comentan, sin saber si es verdad o no lo que la narrativa les expresa. La mejor forma de despertar el interés por el conocimiento es acercar a los 
jóvenes a la investigación histórica y tratándolos como investigadores, que se crean su papel como tal y guiándolos para que puedan ser analíticos, críticos, reflexivos y así propiciar que aprendan a pensar históricamente y sobre todo y más importante, entender su propia realidad, interpretando el pasado.

El desarrollo del pensamiento crítico como culminación del proceso de enseñanza aprendizaje supondrá la formulación de los principales juicios de los alumnos, derivados de su investigación en el aula. Esta fase representa la culminación del proceso de enseñanza-aprendizaje en el que el alumno demuestra la adquisición de un pensamiento crítico, esto se produce cuando, a partir de diversas informaciones, se es capaz de elaborar una teoría y emitir a partir de ella una evaluación y, por lo tanto, una interpretación de carácter autónomo. El docente debe tomar un posicionamiento de falibilidad más que de autoritarismo para "Resaltar la problematicidad e indeterminación de los temas expuestos con el objetivo de favorecer la reflexión en torno a las propias conclusiones obtenidas, así como un respeto crítico e indagativo por la diversidad de planteamientos" (García, 20I9, p. 3I).

Acosta (20I8) refiere que:

En el aprendizaje significativo el alumnado debe analizar, elaborar, manipular y construir para configurar su conocimiento. Este tipo de aprendizaje busca conectar con los intereses del estudiante, a la vez que promueve una práctica duradera. Se trata de una de las reflexiones más sobresalientes que apuesta por el cambio en la orientación de la educación ( $\mathrm{p}$. I7).

Respecto al método científico de las ciencias sociales aplicado en el aula, Torruella y Fernández (20II) refieren que:

En los contextos de enseñanza-aprendizaje, evidentemente, debemos centrarnos en un conocimiento científico de la historia y no en relatos no verificables del pasado. De ahí la importancia de que a los jóvenes estudiantes se les inicie en el trabajo científico siguiendo las mismas pautas que utilizan los 
historiadores. En este sentido, el trabajo sistemático, así como el apoyo a cualquier hipótesis, estudio o conclusión a partir de las fuentes pertinentes, se convierte en un elemento medular del trabajo didáctico (p. 48).

Para promover una de las competencias que potencia el desarrollo del pensamiento histórico, es enseñar a los estudiantes a aprender a manejar la información histórica, bajo una metodología científica. Para lograr favorecer la construcción de dicho pensamiento. "La enseñanza de la historia debe preparar a los alumnos para comprender los problemas humanos reales y frente a éstos tomar posición" (Salazar, 200I, p. 90).

Para llevar a cabo una intervención en el aula y problematizar un tema histórico, se puede emplear la base metodológica de las ciencias sociales que sugiere Prats (20II) y que consiste en los siguientes siete pasos:

I. Planteamiento del problema o cuestión que se quiere estudiar. Delimitación y acotamiento del campo.

2. Estado de la cuestión. Estudio de todas las respuestas o planteamientos que se han hecho en torno al problema.

3. Valoración de las diversas hipótesis formuladas por los autores sobre la base de los elementos internos de cada una de las propuestas.

4. Análisis y clasificación de todas las fuentes primarias y secundarias relacionadas con el problema que se estudia. Este análisis está en función del tipo de fuentes y es muy diferente el tratamiento de una fuente oral que el de una objetual.

5. Fundamentación, si fuere el caso, de la hipótesis del paso anterior. Planteamiento de la problemática en torno a la 174 hipótesis.

6. Análisis demostrativo. Este paso siempre es muy difícil en Historia, ya que los procedimientos para efectuar la demostración dependen del análisis crítico de las fuentes.

7. Redacción de las conclusiones a que se ha llegado. 
Las técnicas que se consideran pertinentes para aplicar el método de aprendizaje por descubrimiento en el aula son las que sugieren Prats y Santacana (20II). Según los autores del libro Didáctica y Geografía de la Historia, son varios los formatos que se pueden emplear para trabajar dicho método: a) Situación problema; b) Estudio de caso único; c) Trabajo por proyectos; d) Juegos de simulación; e) Dramatizaciones o simulaciones.

\section{Una aproximación a la técnica de trabajo por proyectos}

$\mathrm{Su}$ antecedente cercano al presente es el proyecto History I3-I6, desarrollado en Inglaterra, y es uno de muchos que se fundamentaron en el método por descubrimiento, el cual considera que para que la historia sea relevante en el aula ha de responder a las necesidades personales y sociales del adolescente. Dicho proyecto asume que la única forma de que el conocimiento histórico tenga una base racional y pueda ser comprendida por el alumno, es centrarse en la presentación de las perspectivas, la lógica y los métodos de disciplina histórica. Este proyecto fue producto de la reflexión sobre la naturaleza del conocimiento histórico y los problemas que implica su enseñanza. Se pretendió que la enseñanza de la historia debía apuntar hacia la comprensión del método para analizar el pasado, mediante una aplicación de destrezas y habilidades del alumno sobre un contenido específico.

La técnica de trabajo por proyectos se desprende del método de aprendizaje por descubrimiento. "Es una estrategia de enseñanza con la perspectiva situada y experiencial” (Díaz, 2006, p.28). El aprendizaje por descubrimiento es un tipo de aprendizaje que se entiende como una actividad autorreguladora de investigación, a través de la resolución significativa de problemas, que requiere la comprobación de hipótesis como centro lógico del descubrimiento. Para no constituir un descubrimiento fortuito, el resultado producido ha de conllevar un cambio relativamente estable en la competencia del sujeto. 
Sobre el enfoque de trabajo por proyectos en el aula, Díaz (2006) refiere lo siguiente:

El enfoque de proyectos asume una perspectiva situada en la medida en que su fin es acercar a los estudiantes al comportamiento propio de los científicos sociales destacando el proceso mediante el cual adquieren poco a poco las competencias propias de éstos, por supuesto en sintonía con el nivel educativo y las posibilidades de alcance de la experiencia educativa (p. 33).

"Las fuentes se deben contextualizar con el proyecto de investigación que parte de un tema y se deben relacionar con los tres tipos de contenidos (...), convirtiéndose en una estrategia imprescindible desde el punto de vista didáctico" (Hernández, 2008, p.Io). El desarrollo de competencia "Manejo de la información histórica" permite movilizar conocimientos, habilidades y actitudes para seleccionar, analizar y evaluar críticamente fuentes de información, así como expresar puntos de vista fundamentados sobre el pasado.

\section{Conclusiones}

Los historiadores/investigadores, se apoyan de metodologías para indagar la realidad social, así como fenómenos y objetos de estudio específicos. Su investigación se nutre de los enfoques o paradigmas cualitativo, cuantitativo y comparativo. Cada uno de ellos tiene sus características muy particulares, así como las técnicas y los instrumentos que deben adoptar los historiadores para llevar a cabo una investigación, bajo el método científico, para llegar a la verificación

176 de sus hipótesis.

Mientras que la investigación cualitativa intenta hacer una aproximación global de las situaciones históricas sociales para explorarlas, describirlas y comprenderlas de manera inductiva; el predominio del método hipotético-deductivo implica que los científicos sociales focalicen su atención en probar hipótesis a partir de un doble 
referente, por un lado, el cuerpo conceptual, y por el otro la realidad concreta que se estudia.

El enfoque cuantitativo es secuencial y probatorio. Parte de una idea, que va acotándose $\mathrm{y}$, una vez delimitada, se derivan objetivos y preguntas de investigación, se revisa la literatura y se construye un marco o una perspectiva teórica. Este enfoque también se guía por áreas o temas significativos de investigación. Sin embargo, en lugar de que la claridad sobre las preguntas de investigación e hipótesis precede a la recolección y el análisis de los datos. Los estudios cualitativos pueden desarrollar preguntas de investigación e hipótesis antes, durante o después de la recolección y el análisis de los datos.

El enfoque cualitativo busca principalmente "dispersión o expansión" de los datos e información, mientras que el enfoque cuantitativo pretende intencionalmente "acotar" la información (medir con precisión las variables del estudio, tener "foco"). Un estudio cuantitativo se basa en otros previos, el estudio cualitativo se fundamenta primordialmente en sí mismo.

Con el método comparativo se logran establecer semejanzas, diferencias y oposiciones de las variables propuestas por quienes llevan a cabo la investigación para llegar a realidades concretas y pruebas empíricas, construyendo así los referentes empíricos que son componentes fundamentales de la investigación científica. El método comparativo permite describir similitudes y disimilitudes, trabaja con el presente siendo su despliegue horizontal, compara objetos que pertenecen al mismo género, además se apoya en el criterio de homogeneidad y por esta razón se diferencia de la mera comparación.

La utilización del método comparativo en estudios cualitativos en ciencias sociales y en ciencia política, requiere de un investigador que sea prudente en la selección de los casos a comparar, tarea que ha de desarrollar siguiendo criterios metodológicos, y que resulten interesantes de ser contrastadas. La comparación ha devenido en el principal recurso metodológico en la historia de la disciplina; esto ha sido posible por medio de la organización de una cultura metodológica 
compartida por una comunidad científica. En lo que concierne a la metodología comparativa.

La investigación histórica basada en fuentes ayuda al lector, que no hace investigación, a conocer acerca de dichas fuentes sin consultarlas directamente. En el caso de la educación, les permite a profesores y estudiantes contar no sólo con los resultados de la investigación que parte de una problematización y que se orienta con una metodología de la investigación en el aula, sino también con los lugares específicos de donde se obtuvieron dichos resultados; así como con la posibilidad de revisar dichas fuentes para verlas desde otra visión o conocimiento específico, potenciando así el desarrollo de la competencia para el manejo de la información histórica, el desarrollo del pensamiento histórico y las habilidades comunicativas, digitales y creativas, para dar a conocer a manera de producto final los resultados o hallazgos derivados de la investigación.

\section{Referencias}

Acosta, B. (20I8). Aprendizaje significativo y constructivismo. Aprendizaje Significativo y Constructivismo: Características y Metodologías. Campus Educación. Revista Digital Docente, (8). Recuperado de: https://www.campuseducacion.com/revistadigitaldocente/numeros/8/files/assets/common/downloads/Ca mpus\%20Educaci.pdf

Agüera, S. (20I4). El pensamiento histórico: Problemas, competencias y modelos de aprendizaje [Tesis de maestría]. Universidad de Almería, España. Recuperado de: http://repositorio.ual.es/handle/I0835/2809

Álvarez, C. (2008). La etnografía como modelo de investigación en educación. Gazeta de Antropología, 24(I), eio. Recuperado de: http://hdl.handle.net/I048I/6998

Arista, J., y Reynoso, J. (2017). Elementos teórico-disciplinares para desarrollar las competencias históricas en educación secundaria en México. Revista Iberoamericana para la Investigación y el Desarrollo Educativo, 8(I5). Recuperado de: https://www.ride.org.mx/index.php/RIDE/article/view/298 
Bonilla, E., y Rodríguez, P. (1997). Más allá del dilema de los métodos. La investigación en ciencias sociales ( $3^{\mathrm{a}}$ ed.). Santafé de Bogotá: Ediciones Uniandes.

De Almeida, A. (2016). Pesquisa histórica: teoría, metodología e historiografía. Historia, 7(2).

De León, C., y de la Garza, E. (2013). Método Comparativo. Recuperado de: http://eprints.uanl.mx/9802/I/Estudio\%20Comparado.pdf

Denzin, N. K., y Lincoln, Y. S. (1994). Introduction: Entering the field of quali-tative research. In N. K. Denzin y Y. S. Lincoln (eds.), Handbook of Qualitative Research. Thousand Oaks, California: Sage.

Díaz, F. (2006). Enseñanza situada: vínculo entre la escuela y la vida. México: Mc Graw-Hill Interamericana.

García, D. (2019). Aprendizaje significativo: Aplicación en la enseñanza de la Historia. Campus Educación. Revista Digital Docente, (I3). Recuperado

de:

https://www.campuseducacion.com/blog/revista-digitaldocente/el-aprendizaje-significativo/

Gómez, C., Miralles, P., y Molina, S. (2015). La evaluación del pensamiento histórico en la enseñanza de las ciencias sociales. Contribución al desarrollo de la competencia social y ciudadana. Revista de Estudios Sociales, (52), 9-I4. Recuperado de https://journals.openedition.org/revestudsoc/8992

Grajales, T. (2002). La metodología de la investigación histórica: una crisis compartida. Enfoques, XIV(I), 5-2I. Recuperado de: https://www.redalyc.org/pdf/259/259I4IO4.pdf

Hernández, F. (2008). Didáctica de las ciencias sociales, geografía e historia. México: SEP / Editorial Graó.

Hernández, R., Baptista, P., y Fernández, C. (20I0). Metodología de la instigación ( $5^{\mathrm{a}}$ ed.). México: McGraw-Hill.

Hurtado, J. (2008). Guía para la comprensión Holística de la ciencia. Recuperado http://virtual.urbe.edu/tesispub/o092769/capo3.pdf

Hurtado, J. M. (s/f.). Fuentes primarias y secundarias en la construcción del conocimiento histórico. Recuperado de: http://www.dgespe.sep.gob.mx/public/comunidades/historia/re cursos/Fuentes\%2oPrimarias\%20Hurtado.pdf 
Monje, C. (20II). Metodología de la investigación cuantitativa y cualitativa. Guía didáctica. Colombia: Universidad Surcolombiana / Facultad de Ciencias Sociales y Humanas.

Nohlen, D. (I988). Método comparativo. En R. Reyes (ed.), Terminología Científico-Social. Madrid: Anthropos.

Nohlen, D. (2003). El contexto hace la diferencia. México: C. Zilla / UNAM. Recuperado de: http://ru.juridicas.unam.mx/xmlui/handle/I23456789/10054

Prats, J., y Santacana, J. (20II). Métodos para la enseñanza de la Historia. En Didáctica de la Geografía y la Historia. Formación del profesorado. Educación Secundaria. España: Ministerio de Educación / Editorial Graó. Recuperado de: https://sede.educacion.gob.es/publiventa/PdfServlet?pdf=VPI4 883.pdf\&area $=\mathrm{E}$

Prats, J. (20II). Geografía e Historia. Investigación, innovación y buenas prácticas. Educación Secundaria. España: Ministerio de Educación / Editorial Graó. Recuperado de: https://dialnet.unirioja.es/servlet/libro? codigo $=466833$

Salazar, J. (200I). Problemas de enseñanza y aprendizaje de la Historia. ¿...Y los maestros qué enseñamos por Historia? [Colección Educación, núm. Io]. México: Universidad Pedagógica Nacional. Recuperado de: http://xplora.ajusco.upn.mx:8080/xplorapdf/Julia\%20Salazar\%20Sotelo.pdf

Santisteban, A. (20I0). La formación de competencias de pensamiento histórico. Clío \& Asociados. Memoria Académica, (I4), 34-56. Recuperado de: http://www.memoria.fahce.unlp.edu.ar/art_revistas/pr.40I9/pr. 40I9.pdf

SEP [Secretaría de Educación Pública]. (20IIa). Enseñanza y aprendizaje de la Historia en Educación Básica. México: SEP. Recuperado de http://www.ub.edu/histodidactica/images/documentos/pdf/ens enanza_aprendizaje historia_educacion_basica.pdf

SEP (20IIb). Programas de estudio 20II. Guía para el Maestro. Educación Básica. Secundaria. Historia. México: SEP. Recuperado de https:/coleccion.siaeducacion.org/sites/default/files/files/histor ia_20II.pdf

SEP (20I7). Aprendizajes clave para la educación integral. Plan y programas de estudio para la educación básica. México: SEP. Recuperado de 
https://www.planyprogramasdestudio.sep.gob.mx/descargables /biblioteca/secundaria/historia/I-LpM-sec-Historia.pdf

Tonon, G. (20II). La utilización del método comparativo en estudios cualitativos. En Ciencia Política y Ciencias Sociales. Kairos: Revista de Temas Sociales, I5(27), I-I2. Recuperado de: https://dialnet.unirioja.es/servlet/articulo?codigo $=3702607$

Torruella, F., y Fernández, F. (20II). I2 ideas clave. Enseñar y aprender Historia. España: Editorial Graó. Recuperado de: https:/educacionhistoricabenm.files.wordpress.com/20I5/I2/I2i deasclaveensearyaprenderhistoriapdf-I40331I85502phpappo22.pdf

Este artículo se publica bajo una licencia de Creative Commons Reconocimiento-NoComercial 4.0 Internacional, y puede ser usados gratuitamente para fines no comerciales, dando los créditos a los autores y a la revista. 